Cardiopulmonary

\title{
Support
}

and Physiology

\section{Clinical experience with 202 adults receiving extracorporeal membrane oxygenation for cardiac failure: Survival at five years}

Nicholas G. Smedira, MD

Nader Moazami, MDa

Camille M. Goldinga

Patrick M. McCarthy, MD

Carolyn Apperson-Hansen, MStat ${ }^{b}$

Eugene $\mathrm{H}$. Blackstone, $\mathrm{MD}^{\mathrm{a}, \mathrm{b}}$

Delos M. Cosgrove III, MDa

From the Department of Thoracic and Cardiovascular Surgery ${ }^{\mathrm{a}}$ and the Department of Biostatistics and Epidemiology, ${ }^{\mathrm{b}}$ The Cleveland Clinic Foundation, Cleveland, Ohio.

Read at the Eightieth Annual Meeting of The American Association for Thoracic Surgery, Toronto, Ontario, Canada, April 30-May 3, 2000.

Received for publication May 4, 2000; revisions requested Aug 1, 2000; revisions received Jan 8, 2001; accepted for publication Jan 17, 2001.

Address for reprints: Nicholas G. Smedira, MD, The Cleveland Clinic Foundation, 9500 Euclid Ave, Desk F25, Cleveland, OH 44195 (E-mail: smedirn@ccf.org).

J Thorac Cardiovasc Surg 2001;122:92-102

Copyright (C) 2001 by The American Association for Thoracic Surgery

$0022-5223 / 2001 \$ 35.00+0 \quad \mathbf{1 2 / 6 / 1 1 4 3 5 1}$

doi: $10.1067 / \mathrm{mtc} .2001 .114351$
Objectives: We sought to determine 5-year survival after extracorporeal membrane oxygenation for cardiac failure and its predictors, to assess survival and its predictors after bridging to transplantation or weaning from extracorporeal membrane oxygenation, and to identify factors influencing the likelihood of these outcomes.

Methods: Two hundred two adults (mean age, $55 \pm 14$ years) were supported with extracorporeal membrane oxygenation between 1992 and July 1999 after cardiac failure. Follow-up extended to 7.5 years (mean, $3.8 \pm 2$ years). Multivariable hazard function analysis identified predictors of survival, and logistic regression identified the determinants of bridging or weaning.

Results: Survival at 3 days, 30 days, and 5 years was $76 \%, 38 \%$, and $24 \%$, respectively. Patients surviving 30 days had a $63 \% 5$-year survival. Risk factors $(P<.1)$ included older age, reoperation, and thoracic aorta repair. Forty-eight patients were bridged to transplantation, and 71 were weaned with intent for survival. Survival was similar after either outcome (44\% vs 40\% 5-year survival, respectively). Failure to bridge or wean included $(P<.03)$ renal and hepatic failure on extracorporeal membrane oxygenator support, occurrence of a neurologic event, and absence of infection. The dominant modes of death were cardiac failure and multisystem organ failure.

Conclusions: Extracorporeal membrane oxygenation is versatile and salvages some patients who would otherwise die. Improvement in intermediate-term outcome will require a multidisciplinary approach to protect organ function and limit organ injury before and during this support.

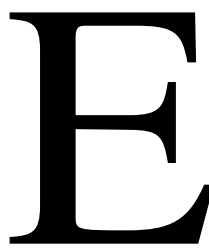

xtracorporeal life support (extracorporeal membrane oxygenation [ECMO]) in newborns, infants, and children has been widely reported to have excellent results. ${ }^{1,2}$ ECMO in adults is less widely practiced and has met with less success. The focus of most reports has been on events during and shortly after ECMO. 
Evolving from the experience with postcardiotomy centrifugal pump support at The Cleveland Clinic Foundation, ECMO has been used for circulatory support in a relatively large number of adults. The purpose of this study was primarily to determine intermediate-range (5-year) survival and its pre-ECMO predictors. Secondarily, survival and its predictors were assessed separately among patients bridged from ECMO to transplantation or weaned from ECMO with the intent of survival. To achieve the secondary purpose, we also identified both pre-ECMO and ECMO factors that were related to whether a patient was bridged to transplantation, weaned from ECMO, or withdrawn from ECMO because of futility.

\section{Patients and Methods Patients}

Between 1992 and July 1, 1999, 202 patients were supported with ECMO after cardiac failure. Patients with right ventricular failure after insertion of an implantable left ventricular assist device (LVAD) supported with ECMO as a right ventricular assist device were not included in the analysis nor were patients supported by venovenous ECMO for respiratory support. Patients supported with ECMO ranged in age from 18 to 82 years (mean, $55 \pm 14$ years), and 145 (72\%) were men. The general clinical characteristics of these patients are presented in Appendix Tables A and B.

Of the 202 patients, 107 (53\%) had undergone cardiotomy (Appendix Table C). Among the patients receiving ECMO after cardiotomy, $60(56 \%)$ had undergone an isolated coronary artery bypass grafting procedure, $19(18 \%)$ had undergone coronary artery bypass grafting and a valve operation, 15 (14\%) had undergone a valve operation, 8 (7\%) had undergone repair of congenital heart disease, 4 (4\%) had undergone aortic aneurysm repair, and 1 (1\%) had undergone left ventriculectomy.

\section{Conduct of ECMO}

ECMO was initiated at the discretion of the operating or transplant surgeon or interventional cardiologist, usually within 30 to 45 minutes of deciding to support the patient. For postcardiotomy patients, failure to wean from bypass on inotropic agents and intraaortic balloon pump (IABP) support prompted ECMO insertion. Absolute hemodynamic criteria were not used. For patients in shock, ECMO was preferred over direct LVAD support because many patients had prior cardiac operations, were near death, and candidacy for transplantation had not been clarified.

Venoarterial ECMO was instituted by means of peripheral cannulation (common femoral artery and vein) in $153(76 \%)$ patients and central cannulation (ascending aorta and right atrium or common femoral vein) in 49 (24\%) patients.

The ECMO circuit and cannulation protocols have been described in detail previously. ${ }^{3,4}$ In brief, the circuit is based on a centrifugal blood pump (Bio-Pump BP-80; Medtronic BioMedicus, Eden Prairie, Minn) in conjunction with an oxygenator (Maxima Plus PRF, Medtronic Cardiac Surgery; Medtronic, Inc, Anaheim, Calif). All components are heparin coated. The patient is heparinized as early as possible to attain an activated clotting time of 180 to 250 seconds.
All patients were supported with the intention to wean from ECMO. Intravenous inotropic doses were reduced but continued at a lower level to maintain ventricular ejection. IABP support was encouraged while patients received ECMO. Atrial septostomy or ventricular venting was not used. Recovery was assessed daily by means of clinical, hemodynamic, and echocardiographic findings. When recovery was unlikely or uncertain, transplant screening was initiated. Candidacy for transplantation and LVAD bridge was made on a case-by-case basis. The only absolute contraindication was profound neurologic injury, which was usually corroborated by computed tomographic scan, and severe active infection. Mildto-moderate encephalopathy, elevated bilirubin and creatinine levels, and treated intravenous line and pulmonary infections did not exclude a patient from bridge-to-transplant consideration. In patients with absolute contraindications to transplantation, weaning was performed with the intent for survival. ECMO was withdrawn from some patients with the knowledge that survival was unlikely and continued support was futile.

\section{Follow-up}

Because the focus of this study was on time-related survival, all patients were followed up as of March 2000. At that time, the range of follow-up time among the 51 patients still alive was 1.1 to 7.5 years (mean, $3.8 \pm 1.62$ years). Among the 151 patients who died, median survival was 5 days, and 142 (94\%) of the 151 deaths were within the first 6 months.

\section{Methods of Data Analysis}

Descriptive. Descriptive statistics are summarized as means and SDs for continuous variables when they were approximately normally distributed but as medians, 25 th and 75th percentiles, and ranges when they were not. Categorical variables are expressed as percentages.

Analytic strategy. The primary end point for this study was death at any time after the start of ECMO. Secondarily, we examined time-related survival after weaning from ECMO with the intent for survival $(n=71)$ or bridge to transplantation $(n=48)$. This secondary aim required that we also identify the predictors of bridging, weaning, or withdrawal of ECMO life support.

Nonparametric estimates of survival were obtained by following the method of Kaplan and Meier. ${ }^{5}$ A parametric method was used to resolve the number of phases of instantaneous risk of death (hazard function) and to estimate their shaping parameters. ${ }^{6 *}$ For the primary end point of survival, time zero was taken as the start of ECMO. For the secondary analysis of survival after bridging or weaning, time zero was taken as the time the patient was removed from ECMO.

Multivariable analyses. For the primary multivariable analysis of time-related survival, the question being answered was as follows: What are the factors associated with time-related survival that are known at the time of decision making for ECMO? Thus, the variables entered into the analysis included demography, cardiopulmonary comorbidity, noncardiac comorbidity, the type of cardiac procedure that may have preceded ECMO, and indications for ECMO (Appendix 1).

*Available by anonymous ftp from ftp:Iluabcvsr.cvsr.uab.edu. 
TABLE 1. Complications during ECMO

\begin{tabular}{lcr}
\hline Complication & No. & $\%$ \\
\hline Infections & 99 & 49 \\
Dialysis & 81 & 40 \\
Neurologic events & 66 & 33 \\
Pump thrombus & 12 & 6 \\
Limb complications & 51 & 25 \\
$\quad$ Ischemia* & 37 & 18 \\
Fasciotomy & 4 & 2 \\
Vascular repair & 6 & 3 \\
Amputation & 4 & 2 \\
\hline
\end{tabular}

*Medically treated.

TABLE 2. Highest values during ECMO support

\begin{tabular}{lcccccc}
\hline & & \multicolumn{5}{c}{ Percentile } \\
\cline { 3 - 7 } Variable & $\mathbf{n}$ & Minimum & $\mathbf{2 5}$ & $\mathbf{5 0}$ & $\mathbf{7 5}$ & Maximum \\
\hline BUN (mg/dL) & 170 & 9 & 25 & 40 & 59 & 167 \\
Creatinine (mg/dL) & 173 & 0.6 & 1.5 & 2.2 & 3.3 & 6.5 \\
AST (U/L) & 170 & 1 & 92 & 204 & 475 & 21,000 \\
Total bilirubin & 179 & 0.3 & 1.7 & 3.1 & 8.9 & 103 \\
$\quad(\mathrm{mg} / \mathrm{dL})$ & & & & & & \\
\hline
\end{tabular}

$B U N$, Blood urea nitrogen; $A S T$, aspartate aminotransferase.

For the secondary analyses of survival after bridging or weaning, the question being answered was as follows: What are the preECMO factors associated with survival, and what additional contribution did events during ECMO make? Thus, a sequential analysis was performed, first with pre-ECMO variables as delineated above and then with variables related to events occurring during ECMO and maximal recorded values for measurements made during ECMO (Tables 1 to 3).

Initial screening of variables possibly related to survival used the log-rank test and the Cox proportional hazards model. Continuous and ordinal variables were assessed univariably by means of decile analysis to suggest transformations of scale to incorporate into the multivariable analysis to ensure that the relation of these variables to outcome was well calibrated with respect to model assumptions. For each of the hazard models, the analysis was performed simultaneously for each hazard phase. Variables were identified by a guided technique of entry of variables into the multivariable models. $^{7}$

The $P$ value criterion for retention of variables in the final model was .1. This liberal criterion was chosen because of the possibility that important variables related to survival would be overlooked by too stringent a variable retention criterion as a result of the relatively small number of deaths (type II error). However, this strategy exposed us to the risk of model overdetermination, whereby risk factors cease to be general common denominators and become identifiers of specific individuals in the data set who have died (type I error). Therefore, the low frequency of occurrence variables was not used to balance these
TABLE 3. Neurologic complications during ECMO support

\begin{tabular}{|c|c|c|c|c|c|c|}
\hline \multirow[b]{2}{*}{ Event } & \multicolumn{2}{|c|}{ Weaned $(n=71)$} & \multicolumn{2}{|c|}{ Bridged $(n=48)$} & \multicolumn{2}{|c|}{ Withdrawn $(n=83)$} \\
\hline & $\mathbf{n}$ & $\%$ & $\mathbf{n}$ & $\%$ & $\mathbf{n}$ & $\%$ \\
\hline Encephalopathy & 14 & 20 & 10 & 21 & 23 & 28 \\
\hline Intracranial bleedin & ing 4 & 6 & 1 & 2 & 8 & 10 \\
\hline Stroke & 2 & 3 & 1 & 2 & 3 & 4 \\
\hline Total & 20 & 28 & 12 & 25 & 34 & 41 \\
\hline
\end{tabular}

errors, and all analyses were supplemented with bootstrap resampling variable selection. ${ }^{8,9}$ For this, observations were sampled at random (with replacement) from the data set to generate a new data set $75 \%$ the size of the original. This process was repeated 1000 times. An automated stepwise variable selection was performed (retention criterion, $P=.1$ ) for each bootstrap data set. For clusters of highly correlated variables, such as transformations of the same or similar variables (age and body size), only one member of each cluster was entered. By means of this tactic, the frequency of occurrence of variables within each category yielded an accurate assessment of the likelihood that one member of that cluster should belong in the final model, and it identified which transformation was the most likely one. Thus, the risk factors identified were not only statistically significant but also must have occurred with the highest frequency, as determined with bootstrap analysis. ${ }^{10}$

The final strategy for the analysis was to identify those factors that resulted in patients either being considered unsalvageable (withdrawn, $n=83$ ), bridged ( $n=48$ ), or weaned with intent for survival $(\mathrm{n}=71)$. These were considered competing risks. Therefore, multivariable logistic regression analysis was used with the variables and strategies as stated above but with pairwise conditional groupings of patients, such that in the final analysis the probability of an individual patient belonging to each of the 3 groups added to $100 \% .{ }^{11}$ For these analyses, we sequentially used factors known before ECMO and again after the addition of the factors concerning the complications of and the process of ECMO.

Presentation. Because only $12(6 \%)$ patients have been followed up beyond 5 years, presentation of time-related survival has been truncated at 5 years.

Nonparametric Kaplan-Meier time-related estimates are accompanied by asymmetric confidence limits equivalent to $1 \mathrm{SE} .{ }^{12}$ Parametric estimates are accompanied by confidence limits equivalent to $1 \mathrm{SE} .^{12}$

Tables of risk factors identified in the hazard domain are presented with their regression coefficients rather than hazard ratio because the model is one of nonproportional hazards. Instead, because the hazard function multivariable analyses are completely parametric (generating an equation), nomograms from the analyses are presented in which specific variables, such as age and creatinine level, are entered into the equations, the equations are solved, and the results are presented graphically with confidence limits. This same strategy was used in the logistic regression domain.

All confidence limits presented in this article are equivalent to $1 \mathrm{SE}(68 \%)$ for consistency with the presentation of mean values to $1 \mathrm{SD}$. 


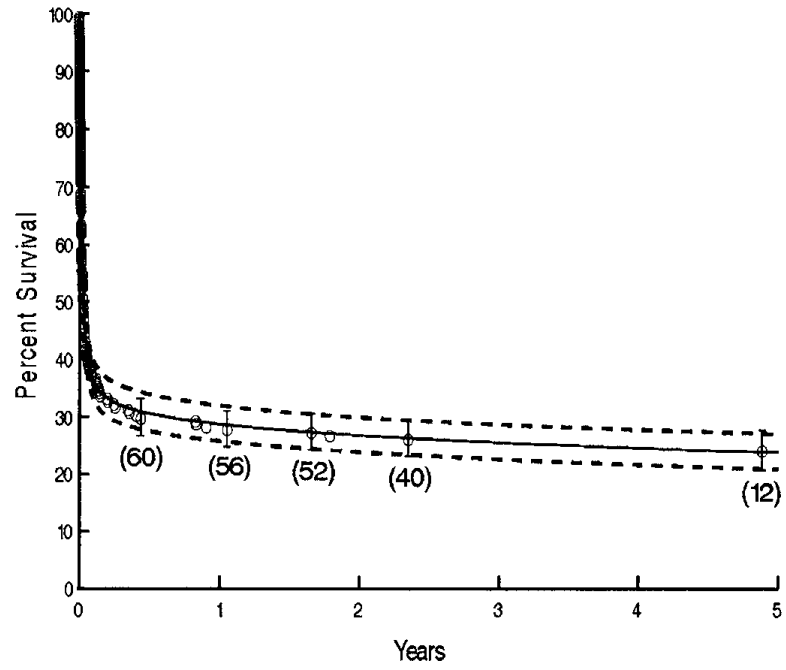

Figure 1. Survival after commencing ECMO (time zero). Each symbol represents a death positioned at the time of death on the horizontal axis and by the Kaplan-Meier estimator on the vertical axis. Periodically, vertical bars represent asymmetric confidence limits equivalent to $1 \mathrm{SE}$. The numbers in parentheses are the number of patients remaining at risk at the time interval shown. The solid lines are parametric estimates enclosed in confidence limits equivalent to $1 \mathrm{SE}$.

\section{Results \\ Survival}

Survival at 24, 48, and 72 hours after initiation of ECMO was $90 \%, 83 \%$, and $76 \%$, respectively. By 1 week, 2 weeks, and 30 days, survival had fallen to $58 \%, 45 \%$, and $38 \%$. By 90 days, 1 year, 3 years, and 5 years, survival was $33 \%, 29 \%$, $26 \%$, and $24 \%$. Patients surviving at 30 days had a $63 \% 5-$ year survival. The instantaneous risk of death had 2 distinct phases: an early high initial rate of death fell to merge at about 1 year with a slowly declining hazard rate (Figure 1).

Pre-ECMO risk factors for death are shown in Table 4. They included older age, with an evident difference in survival above approximately age 55 years (Figure 2). Shorter height was a risk factor that was stronger than female sex. Four patients had prior operations on the thoracic aorta, and all died within 4 days of the start of ECMO. Patients who had a history of an open-chest operation before the present hospitalization leading to ECMO support were at higher risk, as were patients in whom an IABP was not used and patients who were experiencing decompensated heart failure or cardiogenic shock.

\section{ECMO Complications and Values}

Complications occurring while patients were receiving ECMO included infection in $49 \%$, requirement for dialysis in $40 \%$, neurologic events in $33 \%$, and limb complications in $25 \%$ (Table 1). The median transfused units of packed

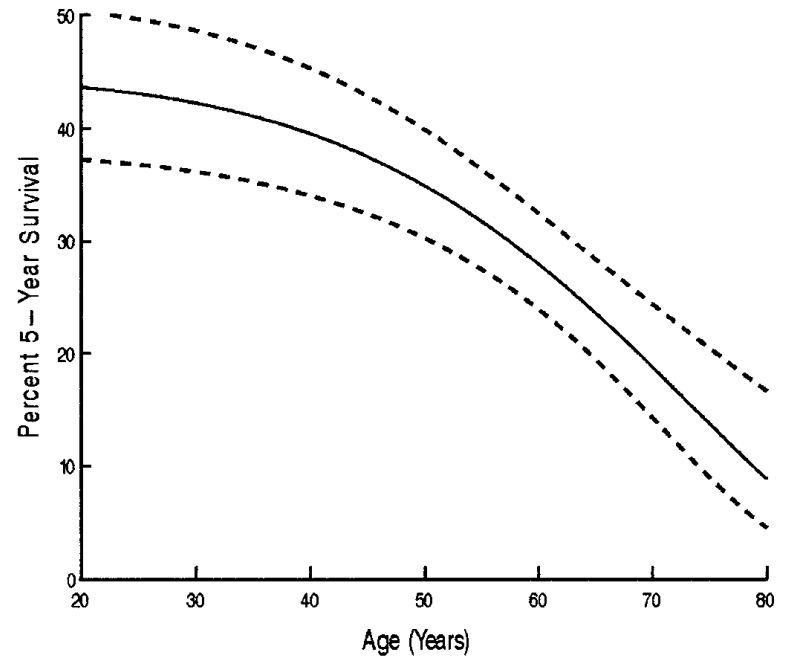

Figure 2. Risk-adjusted relation of age and 5-year survival. This is a so-called nomogram of Table 4, in which height was set to 170 $\mathrm{cm}$, creatinine level was set to $1.5 \mathrm{mg} / \mathrm{dL}$ after a primary nonthoracic aortic operation, no preoperative decompensation was used, and IABP was used.

TABLE 4. Pre-ECMO risk factors for death after initiating ECMO

\begin{tabular}{|c|c|c|}
\hline Incremental risk factors for death & Coefficient \pm SD & $P$ value \\
\hline \multicolumn{3}{|l|}{ Early hazard phase } \\
\hline \multicolumn{3}{|l|}{ Demographic } \\
\hline Older age $\mathrm{e}^{*}$ & $0.29 \pm 0.101$ & .004 \\
\hline Shorter height & $-0.031 \pm 0.0113$ & .006 \\
\hline \multicolumn{3}{|l|}{ Cardiac comorbidity } \\
\hline Primary operationt & $-0.56 \pm 0.26$ & .03 \\
\hline \multicolumn{3}{|l|}{ Procedure } \\
\hline Thoracic aortic operations & $2.1 \pm 0.63$ & .0007 \\
\hline \multicolumn{3}{|l|}{ Late hazard phase } \\
\hline \multicolumn{3}{|l|}{ Cardiac comorbidity } \\
\hline Reoperationt & $1.68 \pm 0.51$ & .001 \\
\hline Nonuse of IABP & $-0.93 \pm 0.41$ & .02 \\
\hline \multicolumn{3}{|l|}{ Indication for ECMO } \\
\hline $\begin{array}{l}\text { Decompensated heart failure- } \\
\text { shock }\end{array}$ & $1.18 \pm 0.46$ & .01 \\
\hline
\end{tabular}

${ }^{*}[\text { age } / 50]^{3}$; cubic transformation.

tThe negative coefficient indicates that patients undergoing reoperation were more likely to survive the early phase of risk, but the positive coefficient in the late hazard phase indicates they were more likely to have poor survival. Thus, in total their death was somewhat protracted in time.

cells was 14 (25th percentile, 8 ; 75 th percentile, 21 ; minimum, 0; maximum, 99).

\section{ECMO Outcomes}

Forty-eight patients were bridged to transplantation, 71 were weaned from ECMO with intent for survival, and 83 


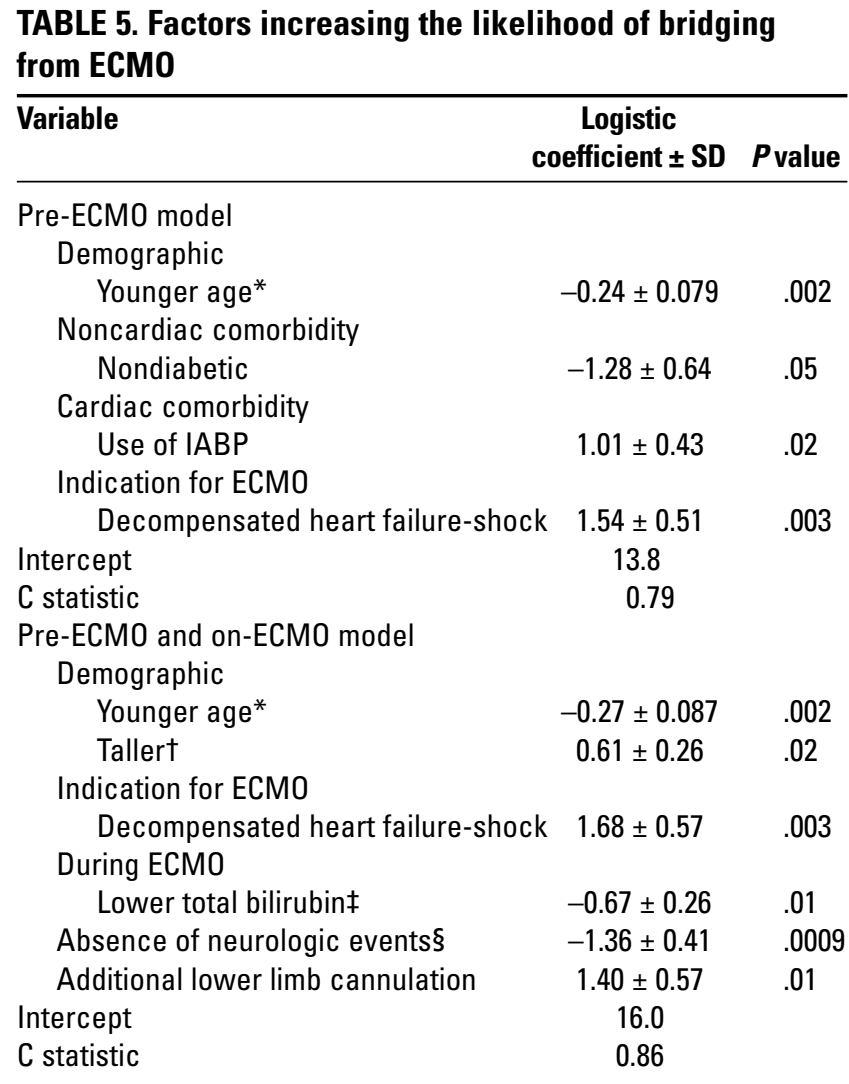

*Uniform age relation to age 60 years and then continuous age thereafter. $+\left[\ln \left[-\ln [\text { height-100]/100] }]^{2}\right.\right.$; normalized squared transformation. ‡ln[total bilirubin]; natural logarithmic transformation.

$\S 0=$ None, 1 = encephalopathy, 2 = stroke; negative coefficient indicates less likelihood of bridging when neurologic events occurred.

were withdrawn from ECMO because of futility. Forty-one percent of those withdrawn from ECMO experienced a neurologic event (Table 3).

Bridged patients. Patients were more likely to be considered for bridging to transplantation if they were younger than 60 years of age and if their heart failure had severely decompensated, leading to ECMO support (Table 5). In addition, nondiabetic patients were more likely to be bridged, as were patients in whom an IABP was used. When events occurring during ECMO and measurements made during ECMO (Tables 1-3) were added to the analysis, lower total bilirubin levels during ECMO, the need for additional lower limb cannulation, and the absence of neurologic events were added (Table 5).

Survival after being bridged from ECMO to an LVAD $(\mathrm{n}=42)$ or directly to transplantation $(\mathrm{n}=6)$ was $85 \%, 67 \%$, $54 \%$, and $44 \%$ at 7 days, 30 days, 1 year, and 5 years, respectively, after cessation of ECMO (Figure 3). Survival to transplantation was $67 \%$, with $92 \%$ of these patients alive 6 months after transplant. Of the 42 patients bridged to an

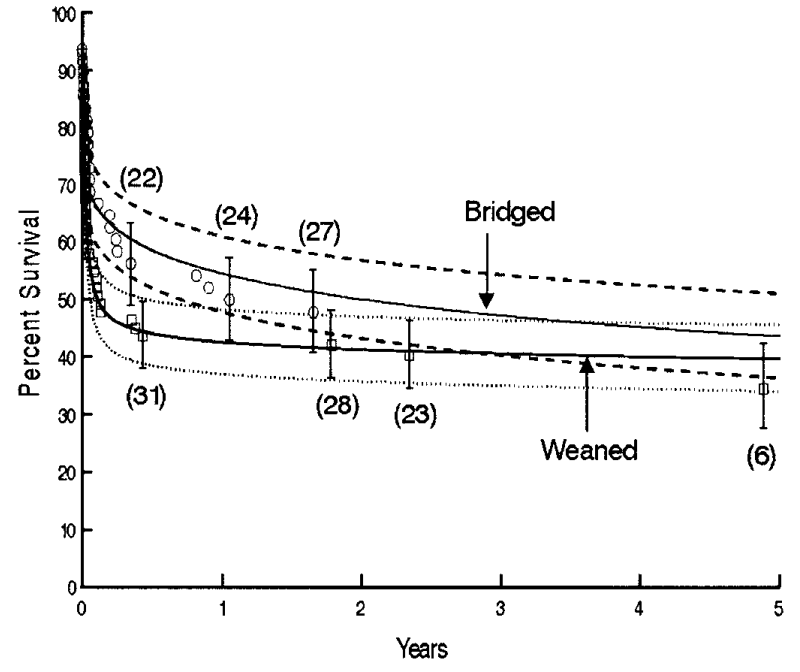

Figure 3. Survival after discontinuing ECMO (time zero) stratified according to the outcome of ECMO: bridged or weaned. Format of the depiction is as for Figure 1.

TABLE 6. Pre-ECMO and on-ECMO factors increasing the likelihood of weaning from ECMO

\begin{tabular}{lcc}
\hline Variable & Logistic coefficient \pm SD & $P$ value \\
\hline Pre-ECMO factors & & \\
$\quad$ Use of IABP & $0.57 \pm 0.33$ & .08 \\
Intercept & -0.48 & \\
C statistic & 0.57 & \\
On-ECMO factors & & \\
$\quad$ Infection & $1.76 \pm 0.42$ & $<.0001$ \\
$\quad$ Lower total bilirubin* & $-0.57 \pm 0.21$ & .008 \\
$\quad$ Lower creatininet & $1.94 \pm 0.84$ & .02 \\
$\quad$ Surgically managed limb & $1.70 \pm 0.81$ & .03 \\
Intercept & -1.37 & \\
C statistic & 0.77 &
\end{tabular}

*In[total bilirubin]; natural logarithmic transformation. $t[1 /$ creatinine $]$; inverse transformation.

LVAD, 25 (60\%) survived to transplantation. The only risk factor for death was older age $(P=.02)$. Patients who had encephalopathy during ECMO experienced reduced survival after being bridged, with 1- and 5-year survivals of $14 \%$ among these 10 patients compared with $61 \%$ and 55\% among the 38 without this complication $(P=.04)$.

Weaned patients. Patients were more likely to be weaned if IABPs were being used (Table 6). Thus, of the 71 patients weaned, 45 (63\%) were receiving pre-ECMO IABP support compared with 41 (49\%) of the 83 withdrawn from ECMO $(P=.08)$. When events during ECMO and measurements were added to the analysis, use of IABP was displaced by 


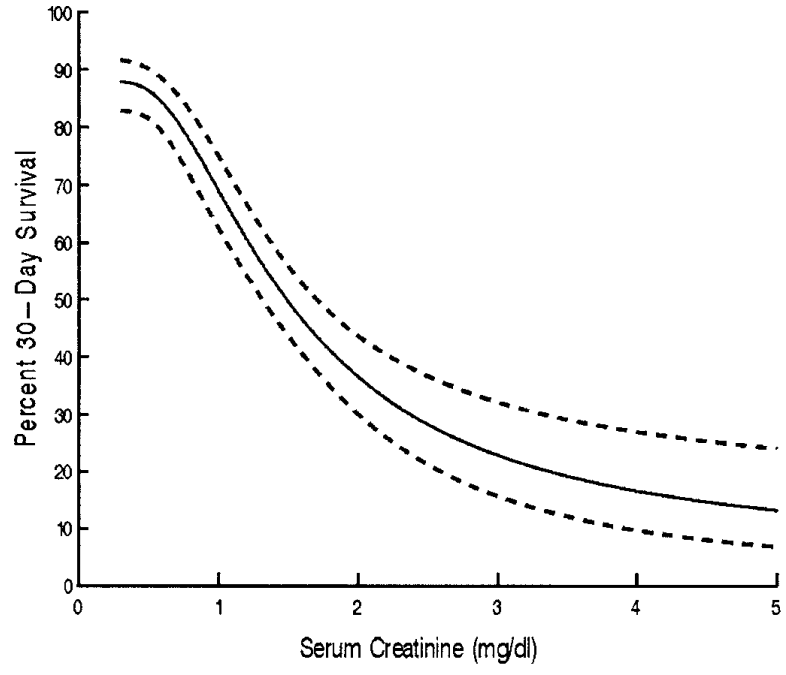

Figure 4. Risk-adjusted relation of $\mathbf{3 0}$-day survival after being weaned from ECMO to pre-ECMO serum creatinine levels. The depiction is a nomogram of Table 7, in which height was set to 170 cm and international normalized ratio to 1.2 .

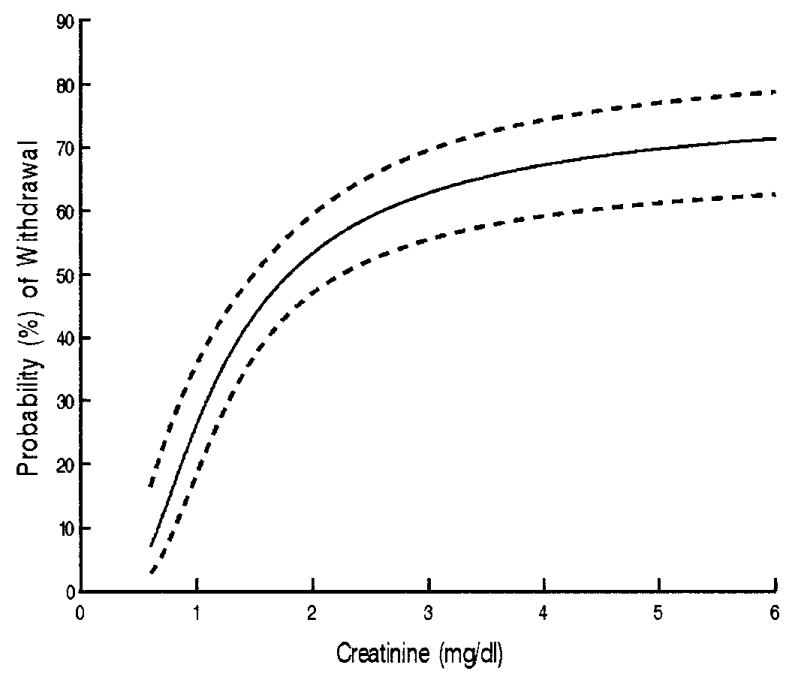

Figure 5. Risk-adjusted relation of ECMO serum creatinine levels to the probability of withdrawal of ECMO. The depiction is as in Figure 4, except bilirubin was set at $3.5 \mathrm{mg} / \mathrm{dL}$, neurologic events were set to "no," pump head clots were set to "no," and infection was set to "no."

them (Table 6). These factors related to less subsystem failure and intensity of treatment of infections.

Survival after being weaned from ECMO was $72 \%, 52 \%$, $43 \%$, and $40 \%$ at 7 days, 30 days, 1 year, and 5 years, respectively, which is similar to that of bridged patients (Figure 3). Survival after weaning was influenced by the pre-ECMO factors of shorter height, higher creatinine lev-
TABLE 7. Risk factor for death after weaning from ECMO with intent for survival

\begin{tabular}{|c|c|c|}
\hline Risk factors for death & Coefficient \pm SD & $P$ value \\
\hline $\begin{array}{l}\text { Early hazard phase } \\
\text { Demographic }\end{array}$ & & \\
\hline $\begin{array}{l}\text { Shorter height* } \\
\text { Noncardiac comorbidit }\end{array}$ & $11.8 \pm 4.0$ & .003 \\
\hline $\begin{array}{l}\text { Higher creatinine } \dagger \\
\text { Late hazard phase }\end{array}$ & $-1.02 \pm 0.28$ & .0003 \\
\hline Higher INR $\ddagger$ & $-2.7 \pm 1.25$ & .03 \\
\hline
\end{tabular}

INR, International normalized ratio.

*[170/height (cm)]; inverse transformation.

$\dagger[2.5 /$ creatinine]; inverse transformation.

$\ddagger[1 / / N R]^{2}$; inverse squared transformation.

TABLE 8. Pre-ECMO factors increasing the likelihood of withdrawal of ECMO

\begin{tabular}{lcc}
\hline Variable & Logistic coefficient \pm SD & $P$ value \\
\hline Pre-ECMO model & & \\
Demographic & & \\
$\quad$ Shorter height* & $-0.28 \pm 0.156$ & .07 \\
Cardiac comorbidity & & \\
$\quad$ Nonuse of IABP & $-0.60 \pm 0.30$ & .04 \\
Intercept & 0.33 & \\
C statistic & 0.60 & \\
On-ECMO model & & \\
Demographic & & .04 \\
Shorter height* & $-0.36 \pm 0.177$ & \\
On-ECMO factors & & .005 \\
Higher creatinine† & $-2.3 \pm 0.83$ & .005 \\
Higher bilirubin & $0.55 \pm 0.193$ & .04 \\
Neurologic events§ & $0.53 \pm 0.25$ & $<.0001$ \\
No infection & $-1.70 \pm 0.38$ & .05 \\
Pump head clots & $1.38 \pm 0.71$ & \\
Intercept & 1.00 & \\
C statistic & 0.77 &
\end{tabular}

*[In [-In[height-100]/100]] $]^{2}$.

$\dagger[2.5 /$ creatinine]; inverse transformation.

fln [total bilirubin]; natural logarithmic transformation.

$\S 0$ = None, 1 = encephalopathy, 2 = stroke.

Negative coefficient indicates less likelihood of withdrawal.

els, and higher international normalized ratio (Table 7). The most powerful was the pre-ECMO serum creatinine level (Figure 4).

Withdrawal from ECMO. Patients withdrawn from ECMO for futility represent the complementary group to bridged and weaned patients. Thus, their pre-ECMO risk factors included shorter height and nonuse of IABP (Table 8). With the addition of factors during ECMO (Table 8), worse renal and hepatic function and complications of 
TABLE 9. Modes of death

\begin{tabular}{|c|c|c|c|c|c|c|}
\hline \multirow[b]{3}{*}{ Mode } & \multicolumn{6}{|c|}{ Outcome } \\
\hline & \multicolumn{2}{|c|}{ Weaned $(n=71)$} & \multicolumn{2}{|c|}{ Bridged $(n=48)$} & \multicolumn{2}{|c|}{ Withdrawn $(\mathrm{n}=83$} \\
\hline & $\mathrm{n}^{*}$ & $\%$ of 34 & $\mathrm{n}^{*}$ & $\%$ of 16 & $\mathbf{n}^{*}$ & $\%$ of 82 \\
\hline Cardiac failure & 16 & 47 & 1 & 6 & 40 & 49 \\
\hline $\begin{array}{l}\text { Multiorgan } \\
\text { system failure }\end{array}$ & 14 & 41 & 14 & 88 & 28 & 34 \\
\hline Neurologic & 4 & 12 & 1 & 6 & 14 & 17 \\
\hline Total deaths & 34 & 100 & 16 & 100 & 82 & 100 \\
\hline
\end{tabular}

*Number of deaths within failure subgroups.

ECMO dominated (Tables 3 and 8). Figure 5 shows the profound influence of higher creatinine levels during ECMO, and Figure 6 shows the influence of higher levels of bilirubin, leading to withdrawal of ECMO. Of the 12 patients in whom pump head clots were found, two thirds were withdrawn from ECMO compared with $39 \%$ of other patients $(P=.06)$.

\section{Modes of Death}

The modes of death differed according to the outcome of ECMO (Table 9). Both those withdrawn from ECMO and those weaned from ECMO died most commonly of cardiac failure, whereas this occurred in only 1 bridged patient; the cause of death was nearly exclusively multiorgan system failure.

\section{Discussion}

ECMO technology is simple to use, is rapidly initiated and applicable to patients of all sizes, and can rapidly reverse ischemia and anoxia. Questions remain as to the utility of this type of support in adult patients and whether the numerous complications previously reported are derived from the insult leading to the need for ECMO or caused, perpetuated, or both by the course of ECMO.

\section{Principal Findings}

Early mortality and morbidity remain very high for patients requiring ECMO support. Older age, evidence of organ system dysfunction either before or during the course of ECMO support, a history of previous cardiac operations, extensive aortic operations, neurologic events, and nonuse of IABP support were variables predictive of death during ECMO or after ECMO was withdrawn. If the patient survived the hospitalization, intermediate-term survival was quite good and was sustained for over 5 years.

The predictors of bridging in part reflect our clinical practice and an aggressive ECMO bridge approach to patients already on the transplant list (severe heart failure

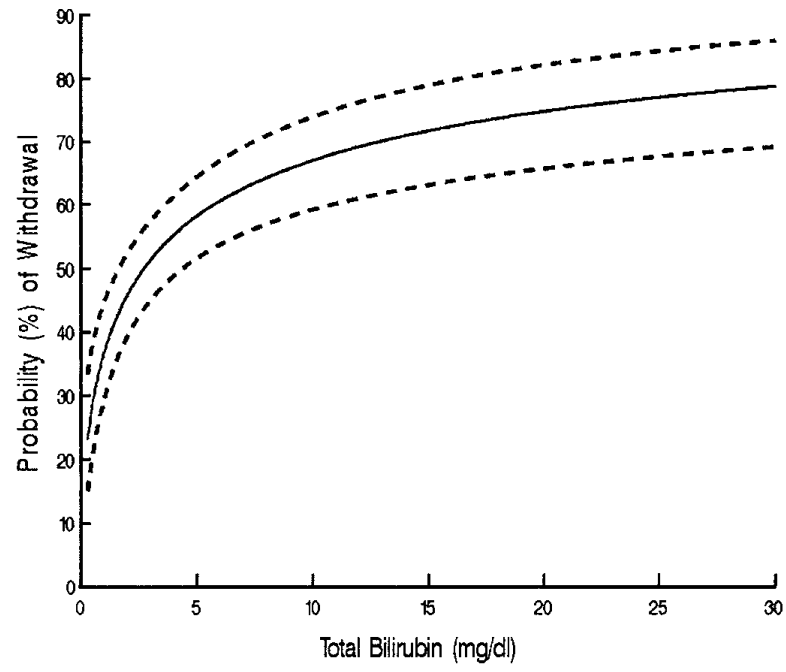

Figure 6. Risk-adjusted relation of ECMO total bilirubin levels to the probability of withdrawal of ECMO. The depiction is as in Figure 5, except serum creatinine level was set to $2 \mathrm{mg} / \mathrm{dL}$.

group). Bridging to transplantation successfully salvaged a sizeable group of young patients who otherwise would have died. Although the survival is less than that of those bridged to transplantation without preceding ECMO support, ${ }^{13}$ it is useful as a temporizing strategy, allowing time for organ recovery and transplant assessment.

\section{ECMO Morbidity and Mortality: The Event or the Device?}

The need for ECMO support occurs after profound cardiopulmonary collapse, failure to wean successfully from cardiopulmonary bypass, and decompensation of chronic congestive heart failure. All these processes are associated with either ischemic or reperfusion organ injury or alterations in the inflammatory response leading to complement, neutrophil, platelet, and cytokine activation. ${ }^{14}$ ECMO produces similar responses, and this undoubtedly compounds the initial insult. ${ }^{15}$ The unanswered question is why this occurs in some patients, but not in others.

Initiating ECMO before complete hemodynamic collapse is ideal, but in most cases the insult has already occurred. Therapies must be developed that alter the reperfusion injury, preserving renal and hepatic function. Agents that affect the inflammatory response in general, such as steroids, aprotinin, and plasmapheresis, or more specific blockades, such as leukocyte depletion or direct cytokine inhibition, will need evaluation in the adult patients undergoing ECMO.

Heparin coating or bonding of the ECMO circuit was used with the hope that it would reduce cellular and mediator activation in addition to the reduction in the anticoagulation requirements. We found that the failure to use heparin resulted in a high occurrence of thrombotic complications 
during ECMO. ${ }^{16}$ Although pump thrombus was uncommon, when it did occur, it was nearly always associated with the patient dying. It has been suggested that microthromboemboli are occurring continuously during ECMO support and contribute to organ injury and dysfunction. ${ }^{17}$ Strategies to reduce these events must be developed. The prolonged use of a centrifugal pump and a microporous membrane oxygenator may have perpetuated cell damage and an inflammatory response. The use of a less traumatic roller pump and silicone membrane oxygenator, as used by the University of Michigan group, may have reduced these complications. However, using this system to bridge patients to an implantable LVAD, Pagani and colleagues ${ }^{14}$ found a 44\% 1-year survival, which is similar to that observed by us.

Current anticoagulation protocols are suboptimal. Although they reduce pump thrombosis, bleeding remains excessive. Undoubtedly, this is multifactorial and includes surgical trauma, anticoagulation, and thrombocytopenia. The use of aminocaproic acid (Amicar) and aprotinin has been advocated with mixed results in neonatal patients. ${ }^{18}$ These agents will need to be used cautiously, weighing the risks of bleeding with those of thrombosis.

Another explanation for the poor early outcome is the absence of pulsatile perfusion. Patients with an IABP were more likely to be weaned or bridged than those without an IABP. This may reflect the beneficial effects of afterload reduction on myocardial recovery or improved organ function with pulsatile flow. Our findings support the recommendation that all patients requiring ECMO support for cardiac failure have concomitant IABP support. ${ }^{19}$ Whether better left ventricular decompression with apical drainage and pulsatility with the BVS 5000 LVAD (Abiomed, Danvers, Mass) would improve myocardial recovery or survival is unclear. The overall postcardiotomy survival reported by Guyton and colleagues ${ }^{20}$ with the BVS 5000 LVAD was only $29 \%$. In addition, the early experience with continuous flow pumping for the bridge to transplantation has been similar to that of the implantable devices, which supports the concept that organ function can be maintained without pulsatility.

\section{Implications for Patient Care}

The majority of patients supported with ECMO were young and justified an aggressive approach. In the older patient survival was markedly reduced, especially in association with pre-ECMO renal or hepatic dysfunction or postECMO neurologic injury. In this group ECMO cannot be advocated, and if ECMO is initiated and rapid clinical improvement is not seen, early termination of ECMO support is warranted.

ECMO provides hemodynamic stabilization and, in the patient with insufficient myocardial recovery, time to assess the patient for transplantation. We aggressively bridged patients from ECMO to an implantable LVAD, with $60 \%$ of the patients surviving to transplantation. The only contraindications for this bridge-to-bridge therapy were severe neurologic injury and active untreated infection. The absence of predictors of survival in this subgroup suggests that some patients with significant alteration in organ function can survive through the double bridge, albeit with a lower survival than in a group of patients with LVADs who are not supported with ECMO.

A substantial number of patients dying during ECMO or after weaning from ECMO died from irreversible myocardial injury. Golding and colleagues ${ }^{21}$ found that $69 \%$ of patients autopsied after postcardiotomy ventricular support had extensive myocardial injury. In the group of patients who are not transplant candidates and who do not have endorgan injury but do have insufficient myocardial recovery, a permanent implantable cardiac assist device would salvage these patients. The ongoing Randomized Evaluation of Mechanical Assistance for the Treatment of Congestive Heart Failure trial will help answer whether the current generation of devices are a durable and cost-effective alternative in this situation. ${ }^{22}$

\section{Limitations}

The analysis of a single clinical experience and the management protocols were evolving over the time period studied. However, results did not change substantially over time and concur with the published results of adult patients receiving ECMO.

\section{Conclusions}

ECMO is versatile and can salvage patients who would otherwise die. Improvement in outcome will require a multidisciplinary approach to protect organ function and limit organ injury that results before and during ECMO support.

\section{References}

1. Bartlett RH, Roloff DW, Custer JR, Younger JG, Hirschl RB. Extracorporeal life support: the University of Michigan experience. JAMA. 2000;283:904-8.

2. Magovern GJ Jr, Simpson KA. Extracorporeal membrane oxygenation for adult cardiac support: the Allegheny experience. Ann Thorac Surg. 1999;68:655-61.

3. McDonald M, Smedira NG. Adult extracorporeal life support. In: Franco K, Verrier E, editors. Advanced therapy in cardiac surgery. Hamilton, Ontario: BC Decker; 1999. p. 326-34.

4. Wudel JH, Hlozek CC, Smedira NG, McCarthy PM. Extracorporeal life support as a post left ventricular assist device implant supplement. ASAIO J. 1997;43:M441-3.

5. Kaplan EL, Meier P. Nonparametric estimation from incomplete observations. J Am Stat Assoc. 1958;53:457-81.

6. Blackstone EH, Naftel DC, Turner ME Jr. The decomposition of timevarying hazard into phases, each incorporating a separate stream of concomitant information. J Am Stat Assoc. 1986;81:615-24.

7. Baskerville JC, Toogood JH. Guided regression modeling for prediction and exploration of structure with many explanatory variables. Technometrics. 1982;24:9-17. 
8. Efron B, Tibshirani R. Bootstrap methods for standard errors, confidence intervals, and other measures of statistical accuracy. Stat Sci. 1986;1:54-77.

9. Altran DG, Andersen PK. Bootstrap investigation of the stability of a Cox regression model. Stat Med. 1989;8:771-83.

10. Breiman L. Bagging predictors. Machine Learning. 1996;26:123-40.

11. Hosmer DW, Lemeshow S. Polytomous logistic regression. In: Applied logistic regression. New York: John Wiley; 1989. p. 216-38.

12. Kirklin JW, Barratt-Boyes BG. Surgical concepts, research methods, and data analysis and use. In: Cardiac surgery. New York: John Wiley; 1986. p. 177-204.

13. Pagani FD, Lynch W, Swaniker F, Dyke DB, Bartlett R, Koelling T, et al. Extracorporeal life support to left ventricular assist device bridge to heart transplant: a strategy to optimize survival and resource utilization. Circulation. 1999;100(Suppl):II-206-10.

14. Taylor KM. Brain damage during cardiopulmonary bypass. Ann Thorac Surg. 1998;65:S20-6.

15. Peek GJ, Firmin RK. The inflammatory and coagulative response to prolonged extracorporeal membrane oxygenation. ASAIO J. 1999;45:250-63.

16. Muehrcke DD, McCarthy PM, Stewart RW, Seshagiri S, Ogella DA, Foster RC, et al. Complications of extracorporeal life support systems using heparin-bound surfaces: the risk of intracardiac clot formation. J Thorac Cardiovasc Surg. 1995;110:843-51.

17. Curtis JJ, Walls JT, Schmaltz RA, Demmy TL, Wagner-Mann CC, McKenney CA. Use of centrifugal pumps for postcardiotomy ventricular failure: technique and anticoagulation. Ann Thorac Surg. 1996; 61:296-300.

18. Horwitz JR, Cofer BR, Warner BW, Cheu HW, Lally DP. A multicenter trial of 6-aminocaproic acid (Amicar) in the prevention of bleeding in infants on ECMO. J Pediatr Surg. 1998;33:1610-3.

19. Lazar HL, Treanor P, Yang XM, Rivers S, Bernard S, Shemin RJ. Enhanced recovery of ischemic myocardium by combining percutaneous bypass with intraaortic balloon pump support. Ann Thorac Surg. 1994;57:663-8.

20. Guyton RA, Schonberger JP, Everts PA, Jett GK, Gray LA, Gielchinsky I, et al. Postcardiotomy shock: clinical evaluation of the BVS 5000 biventricular support system. Ann Thorac Surg. 1993; 56:346-56

21. Golding LA, Crouch RD, Stewart RW, Novoa R, Lytle BW, McCarthy PM, et al. Postcardiotomy centrifugal mechanical ventricular support. Ann Thorac Surg. 1992;54:1059-64.

22. Rose EA, Moskowitz AJ, Packer M, Sollano JA, Williams DL, Tierney AR, et al. The REMATCH Trial: rationale, design, and end points. Randomized Evaluation of Mechanical Assistance for the Treatment of Congestive Heart Failure. Ann Thorac Surg. 1999;67:723-30.

\section{Appendix 1}

\section{Variables Considered in Multivariable Analyses}

- Demographic: age (in years), height (in centimeters), weight (in kilograms), body mass index, body surface area, sex

- Cardiopulmonary comorbidity: reoperation, preoperative use of IABP, duration of ventilation support

- Noncardiopulmonary comorbidity: diabetes mellitus, history of peripheral vascular disease, history of chronic obstructive pulmonary disease, dialysis, blood urea nitrogen (in milligrams per deciliter), serum creatinine (in milligrams per deciliter), aspartate aminotransferase (in units per liter), total bilirubin (in milligrams per deciliter), international normalized ratio (in units), infection

- Preceding operations: coronary artery bypass graft, valve, coronary artery bypass graft and valve, thoracic aortic aneurysm, congenital, lung transplant, heart transplant, left ventriculectomy

- Indications for ECMO: cardiac failure, postcardiotomy, postcardiac catheterization, acute myocardial infarction, acute respiratory disorder, cardiac arrest, myocarditis, postcardiac trans- plant, after lung transplant, decompensated heart failure, postpartum cardiomyopathy, after left ventriculectomy

- Cardiomyopathy variables: dilated cardiomyopathy, ischemic cardiomyopathy

- ECMO details: peripheral cannula used, central cannula used, duration of ECMO, oxygenator count, number of blood transfusions during ECMO, years from January 1, 1992, to ECMO

- ECMO complications: head clot in pump count, additional lower limb cannulation, medically managed limb complication, surgically managed limb complication, dialysis, intracranial bleeding, embolic stroke, encephalopathy, infection

- Highest values during ECMO: blood urea nitrogen (in milligrams per deciliter), serum creatinine (in milligrams per deciliter), aspartate aminotransferase (in units per liter), total bilirubin (in milligrams per deciliter), infection, international normalized ratio (in units)

\section{Discussion}

Dr Robert H. Bartlett (Ann Arbor, Mich). There is a huge amount of data in this article, and it takes hours or days to analyze it, even with the article in hand. I am going to comment on a few of the factors that were raised in this fascinating review.

First, the statistical analysis is superbly done. It is very well described in the article. A tremendous diversity of cardiac and respiratory patients are mixed together in this analysis. What the authors learned, of course, is that older patients (and the oldest was 84 years) and sicker patients with more organ failure tend to die. Well, I guess we all knew that, but it is nice to see it confirmed. You mentioned that older was worse, but you did not tell us what age is "older." Maybe you might comment on that. The subgroup analysis is the really interesting part. A total of 198 patients had partial ventricular assist bypass, almost all of which was femoral-femoral bypass, for cardiac failure, half of which occurred postoperatively and half during cardiogenic shock for some reason; $34 \%$ of those patients survived. One aspect of this article is that death or survival is defined in time increments, and we do not really know when hospital discharge occurred. Therefore, I am assuming they probably were discharged from the hospital if they survived somewhere between 30 and 90 days. Two thirds of the surviving patients (either bridged to spontaneous cardiac recovery or to transplantation) lived for 5 years. This is a very good outcome for those patients, and it begins to define the outcome of the algorithm. About half of the 198 patients receiving ventricular assist bypass were withdrawn from support because they were not candidates for an LVAD or they were not candidates for transplant. However, that is not bad; that is the algorithm. You do the best you can, you get them on bypass, and then you sort things out.

Therefore, with that algorithm, those patients receiving ECMO who have good brain function and reasonable organ function and are transplant candidates go on to LVAD. Their survival was remarkably good: $85 \%$ in this particular group. Our experience is the same.

Thirty-one patients had respiratory failure, and 10 survived. I would advise the authors to write up the respiratory patients separately.

The authors raised the following question: To what extent do the technology and the devices contribute to the mortality? It is a very good question and something we all have to worry about. 


\begin{tabular}{|c|c|c|}
\hline Patient characteristic & No. & $\%$ \\
\hline \multicolumn{3}{|l|}{ Demographic } \\
\hline Men & 145 & 72 \\
\hline \multicolumn{3}{|l|}{ Cardiac comorbidity } \\
\hline Pre-ECMO use of IABP & 115 & 57 \\
\hline Reoperation & 79 & 39 \\
\hline \multicolumn{3}{|l|}{ Noncardiac comorbidity } \\
\hline Diabetes & 42 & 21 \\
\hline Peripheral vascular disease & 30 & 15 \\
\hline COPD & 36 & 18 \\
\hline Chronic dialysis & 7 & 3.5 \\
\hline Infection & 33 & 16 \\
\hline
\end{tabular}

$C O P D$, Chronic obstructive pulmonary disease.

Their technique included the use of a centrifugal pump, which is great for 2 hours of bypass but (in our experience) causes unacceptable amounts of hemolysis and pump thrombus when used for a long time. They used a microporous membrane oxygenator, which undoubtedly leaked plasma and probably had to be changed from time to time. The average activated clotting time was about 200 seconds, and we would usually use much lower than that. Thus, there are issues in the technology that could be changed to improve results even more.

These cases are always emergencies. Maybe you cannot stop bypass in the operating room, or worse yet, it is the middle of the night and your postoperative patient's condition is suddenly deteriorating, or a new patient with a myocardial infarction is admitted with profound cardiogenic shock. My question is, how do you do it? How long does it take you to mobilize the team and get the patient on bypass?

My second question is this: What would you consider to be the contraindications? What patients would you not put on ECMO if you cannot come off bypass in the operating room or have a patient in cardiogenic shock?

Dr Moazami. Dr Bartlett, thank you very much for your comments. We are certainly all aware of your excellent contributions over the years to this technology. I will address your questions.

In terms of "older" patients not doing well, our analysis shows that the inflection point is around 60 years, with patients older than 60 years really not doing well with this technology. I think one of the factors that we tried to highlight in the article, and that I am also trying to emphasize here, is that it is true that the ECMO complications and ECMO factors affect survival, but pre-ECMO factors-in other words, the overall condition of a patient before ECMO is instituted-are also contributing factors. Thus, a patient who has baseline renal insufficiency at the time of insult or hepatic insufficiency actually does not do well with ECMO. If we add these together, I would say a patient who is 65 years old and has a creatinine level of $2 \mathrm{mg} / \mathrm{dL}$ and a low bilirubin level of 2 to 3 $\mathrm{mg} / \mathrm{dL}$ probably has about an $80 \%$ mortality on ECMO.

Your second question referred to how long it takes for us to actually implement the ECMO system. Obviously, we all recognize that the sooner we can resuscitate the patients and put them on ECMO
APPENDIX TABLE B. Patient characteristics before ECMO placement

\begin{tabular}{|c|c|c|c|c|c|c|c|}
\hline \multirow[b]{2}{*}{$\begin{array}{l}\text { Patient characteristic } \\
\text { and general indication }\end{array}$} & \multirow[b]{2}{*}{$\mathbf{n}$} & & \multicolumn{5}{|c|}{ Percentile } \\
\hline & & $\begin{array}{l}\text { Mean } \\
\pm \text { SD M }\end{array}$ & Minimum & n 25 & 50 & 75 & Maximum \\
\hline \multicolumn{8}{|l|}{ Demographic } \\
\hline Age (y) & 202 & $55 \pm 14$ & 18 & 47 & 57 & 65 & 82 \\
\hline Height (cm) & 200 & $171 \pm 9.4$ & 4142 & 1651 & 171 & 178 & 196 \\
\hline Weight (kg) & 200 & $81 \pm 18$ & 42 & 69 & 78 & 90 & 159 \\
\hline \multicolumn{8}{|c|}{ Noncardiac comorbidity } \\
\hline BUN (mg/dL) & 196 & $28 \pm 20$ & 5 & 15 & 22 & 33 & 129 \\
\hline $\begin{array}{l}\text { Serum creatinine } \\
\quad(\mathrm{mg} / \mathrm{dL})\end{array}$ & 196 & - & 0.3 & 1.0 & 1.4 & 1.8 & 11.6 \\
\hline Serum AST (U/L) & 168 & - & 1.4 & 23 & 56 & 181 & 20,300 \\
\hline $\begin{array}{l}\text { Total bilirubin } \\
(\mathrm{mg} / \mathrm{dL})\end{array}$ & 156 & - & 0.05 & 0.8 & $8 \quad 1.1$ & 1.8 & $8 \quad 26.7$ \\
\hline INR (units) & 140 & $1.4 \pm 0.65$ & 50.9 & 1.0 & 1.2 & 1.5 & 5 \\
\hline
\end{tabular}

$B U N$, Blood urea nitrogen; $A S T$, aspartate aminotransferase; INR, international normalized ratio.

APPENDIX TABLE C. Indications for ECMO

\begin{tabular}{lrc}
\hline Indication & No. & $\%$ \\
\hline Postcardiotomy & 107 & 53 \\
Acute MI & 33 & 16 \\
ARDS & 1 & 0.5 \\
Decompensated heart failure & 28 & 14 \\
Cardiac arrest & 12 & 6 \\
After cardiac transplantation & 7 & 3.5 \\
After lung transplantation & 2 & 1 \\
After cardiac catheterization & 7 & 3.5 \\
Myocarditis & 3 & 1.5 \\
Postpartum cardiomyopathy & 1 & 0.5 \\
After left ventriculectomy & 1 & 0.5
\end{tabular}

$M I$, Myocardial infarction; $A R D S$, acute respiratory distress syndrome.

for circulatory support, the better the patients will do. We, as is the case in many other centers, have a mobile system that can be implemented rapidly. The nurses are also very familiar with the management of the system. Although I do not have exact numbers to give you, once it is recognized that ECMO or some other form of support is necessary, the majority of these patients are taken down to the intensive care unit, where support is initiated within 1 hour.

As you mentioned, our approach in $80 \%$ of these patients was peripheral cannulation, which is relatively easy. When we put patients on venovenous bypass for respiratory support, we usually use a percutaneous method based on the Seldinger technique. In patients in whom we use venoarterial support, we primarily do a cutdown in the groin and place the cannulas under direct vision. That also includes placement of a distal perfusion cannula into the superficial femoral artery, which is now a routine practice and has actually cut down significantly on the limb ischemia complications 
that we used to see, particularly those that require a surgical intervention in terms of fasciotomy, revascularization, or even amputation.

In terms of contraindications to ECMO support in the postcardiotomy patients, it is a difficult question, as many of the surgeons who have been faced with this problem would know. If you are in the operating room and you cannot get the patient off bypass, it is difficult to give up. We generally have a graded response to this sequence, which includes maximizing pharmacologic support by addition of inotropic agents (epinephrine and milrinone). Our next step usually is an IABP. Finally, if we cannot get the patient off bypass, then we go to ECMO support.

Dr James Magovern (Pittsburgh, Pa). I just have a couple of brief questions. We also have experience using ECMO for cardiac support. In our experience patients who have a lot of bleeding and require extensive blood transfusion often have multisystem organ failure. Have you seen a relationship between the need for transfusion and subsequent outcome of the patient?

The second issue that we have seen is that patients who recover generally do so within 2 days. The decision, and it becomes an important one, is how long to keep the patient on ECMO. The mean duration of support for survivors in our 10-year experience has been about 44 hours. Therefore, it makes little sense to continue the support past 3 or 4 days if the patient has not recovered function by then. My second question is this: What is the time period under which you see recovery, and when would you give up and stop the ECMO?

Dr Moazami. Dr Magovern, we also see a significant amount of bleeding complications from ECMO support, to which you have alluded. In our patient group there was a large range of blood transfusions, anywhere from 1 to 99 units, but overall, our median transfusion was 13 units per patient. We did not identify transfusion as a risk factor for development of multisystem organ failure. I am aware that this is certainly a key factor, specifically in patients who are receiving assistance from support devices.

In terms of how long we keep the patients on ECMO, our protocol generally is to evaluate the patients within 24 hours to see whether they can be weaned from support. At the same time, we also initiate evaluation for whether the patient is a transplant candidate. During this 24 hours, as I mentioned, we optimize pharmacologic support and subsequently assess whether the patient can be weaned from support by using a combination of clinical parameters; looking at the pulmonary artery pressures, the cardiac output, and the mixed venous saturations; and using transesophageal echocardiography.

Our median duration of support for the cardiac failure group was $2 \frac{1}{2}$ days, which is very close to what you are suggesting, and usually, at that point, we have a clear idea whether we can bridge the patient to an LVAD as a more long-term form of support, whether the patient can be weaned from support, or whether we should withdraw support.

Dr Shyam K. Kolvekar (London, England). We have extensive experience in ECMO, and I just wanted to know who manages your ECMO in the intensive care unit. The second question mainly concerns your bleeding problem in transfusion and whether you have bleeding from your operative wounds or the cannulations.

Dr Moazami. The initial management of the ECMO system is by the physicians and perfusionists; however, the day-to-day and hour-to-hour management is done by our intensive care unit nurses, who have been trained in the management of ECMO and can recognize problems that may arise and notify the appropriate personnel. We usually have a perfusionist in house who can respond quickly, and obviously, the physicians are available.

In terms of your second question, we notice bleeding from the cannulation site, and in some cases in which we have placed the cannulas in the operating room, bleeding can obviously come from the mediastinum. There have been occasions in which we had to pack the chest open and re-explore later.

Dr Marko I. Turina (Zurich, Switzerland). You are surely aware that ECMO is rapidly being developed as a very efficient method for postoperative support in newborns and patients with congenital heart disease after complex reconstructions with results that are definitely better than those seen in adults. Do you have any idea whether it is the disease or the system and why it works better in newborns and in children for postoperative support?

Also, do you routinely decompress the left ventricle in these cases?

Dr Moazami. In response to your first question, I think the indications for placement of neonatal patients on ECMO are quite different from what we see in the adult population; generally, most of them are receiving respiratory support, and the most excellent results have been reported in that subgroup of patients.

In terms of your second question, whether we use any kind of left ventricular decompression, we do not employ such decompression. It is a recognized fact that ECMO support can increase the afterload. I believe that the Michigan group, in some instances when they think there is increased afterload, performs an atrial septostomy. In some cases apical ventriculectomies have been reported, but we do not use those techniques. However, we do, as I mentioned earlier, keep the patients on inotropic support and maintain ventricular ejection during this period. Part of the reason is because of the issue with ventricular distention, but part of it is also because we are always worried about stasis in the left ventricle and formation of intraventricular thrombosis. 